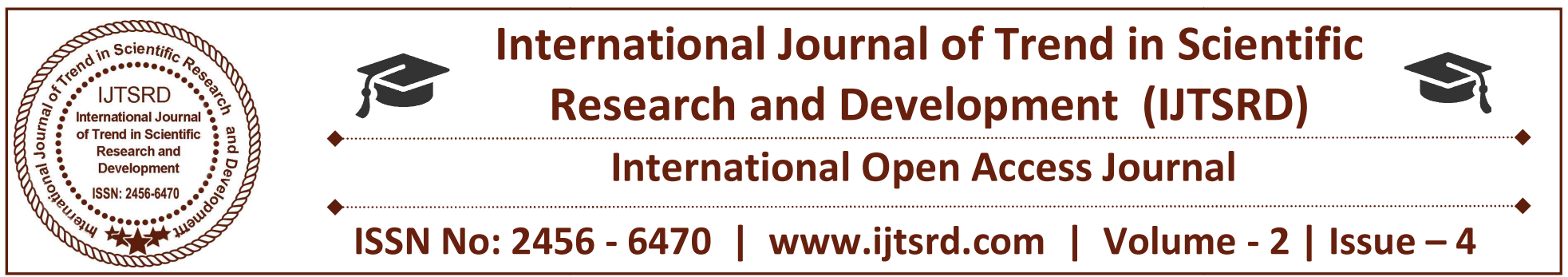

\title{
An Image Quality Assessment of Multi-Exposure Image Fusion by Improving SSIM
}

\author{
Sushmitha $\mathbf{C M}^{1}$, Meharunnisa $\mathbf{S P}^{2}$ \\ ${ }^{1}$ Student, ${ }^{2}$ Assistant Professor \\ Dept of Electronics and instrumentation Engineering, Dayananda Sagar College of Engineering, \\ Bengaluru, Karnataka, India
}

\begin{abstract}
Multi-exposure image fusion (MEF) is viewed as a viable quality upgrade system generally received in purchaser hardware, A solitary caught picture of a genuine scene is normally lacking to uncover every one of the points of interest due to under-or overuncovered locales. In this paper a multi-presentation picture fusion(MEF) calculation by streamlining a target quality measure in particular the MEF auxiliary similitude list (MEF-SSIM). In particular, first develop the MEF-SSIM list by enhancing and growing the application extent of the current MEFSSIM calculation. The last superb picture has little reliance on introductory picture. Trial comes about exhibit the predominance of the proposed technique as far as subjective and target assessment.
\end{abstract}

Keywords: Multi-exposure image fusion(MEF), structural similarity(SSIM)

\section{INTRODUCTION}

The picture combination is one of the vital branches of information combination. Information combination methods has been intended to permit mix of various data sources and furthermore to exploit reciprocal. There is no special definition for picture combination, Few picture combination definitions are given beneath: Image combination is the mix of at least two distinct pictures to shape another picture by utilizing a specific calculation (Genderen and Pohl 1994)[1].Image combination is the way toward joining data from at least two pictures of a scene into a solitary composite picture that is more educational and is more appropriate for visual discernment or PC preparing. (Visitor article of Information Fusion,
2007)[2]. Picture combination is a procedure of consolidating pictures, got by sensors of various wavelengths all the while review of a similar scene, to shape a composite picture. Multi-presentation picture combination (MEF) is viewed as a powerful quality improvement procedure that is generally received in purchaser gadgets [3]. Images taken by ordinary digital cameras usually suffer from lack of details in the under-exposed and over-exposed areas if the camera has a low or high exposure setting, High dynamic range (HDR) imaging solves this problem by taking multiple images at different exposure levels and merging them together, This technique has been widely used in digital camera and mobile phones. Existing HDR imaging approaches can be divided into two categories: tone mapping based methods and image fusion based methods[4]. Multi exposure image fusion (MEF) is a cost effective technique that bridges the gap between the high dynamic range (HDR) of luminance levels in natural scenes and the low dynamic range (LDR) of standard display devices [5].

The info grouping of MEF calculation comprises of numerous photos of a similar scene that is a picture succession taken at various presentation levels, each picture which catches incomplete data of the scene. Most existing multi-introduction combination techniques fundamental supposition is that the scene is static amid various catches. While intertwining pictures taken in powerful scenes which contain camera development or movement questions, the techniques specified above may deliver genuine bends [4]. To expel the effects of camera development, 
numerous multi presentation picture arrangement strategies have been proposed [6]. There are numerous calculations have been proposed as of late, none of them has been intended to improve a guarantee quality measure that relates well with human visual recognition. For instance, a normally utilized approach is to augment the fine subtle elements in combined pictures as an approach to make striking appearance [7], [8]. In addition, every single existing calculation begin by pre-characterizing an efficient computational structure for MEF (e.g., multidetermination change and change area combination took after by picture remaking), with frail and backhanded help of the legitimacy and optimality of such a structure[9]. What's more, most existing MEF calculations are shown utilizing a predetermined number of hand-picked cases, without subjective confirmations on databases that contain adequate varieties of picture substance or target appraisal by settled and subject-approved quality models [10].

Multi-determination changes have been perceived as an exceptionally valuable strategy to examine the data substance of pictures with the end goal of picture combination. The discrete wavelet change has turned into an exceptionally helpful apparatus for combination. These techniques demonstrate a superior execution in spatial and phantom nature of the melded picture. An element solidly identified with picture quality is main focus. A sharp picture gives preferable data over obscure pictures. At any rate, in a few circumstances it isn't conceivable to get totally engaged pictures in only one single camera shot, since a few areas seems, by all accounts, to be obscured as a result of varieties in the profundity of the scene and because of the camera focal points center. This implies if the camera is engaged at one particular point, another area of the scene can be out of core interest. A fascinating arrangement is that to take more photos of the coveted scene similarly situated, however with center focused in various components of the protest. At that point utilizing the picture combination idea all source pictures are joined, by making a solitary picture that contains all the ideal centered locales. Picture combination is winding up exceptionally prevalent in advanced picture handling. The UIs, models and menus accessible with MATLAB GUIDE, and are utilized for simple usage of calculations and to limit code length, conceivable events of blunders with calculations.

\section{RELATED WORK}

Shutao Li and Xudong Kang [11] proposes a weighted total based multi-presentation picture combination technique which comprises of two primary advances: three picture highlights made out of nearby difference, brilliance and shading divergence are first estimated to assess the weight maps refined by recursive sifting. At that point, the intertwined picture is developed by weighted whole of source pictures. The fundamental favorable position of the proposed strategy lies in a recursive channel based weight delineate advance which can acquire precise weight maps for picture combination. Another favorable position is that a novel histogram balance and middle channel based movement discovery strategy is proposed for combining multi-introduction pictures in unique scenes which contain movement objects. Besides, the proposed strategy is very quick and in this way can be straightforwardly utilized for most purchaser cameras. Trial comes about exhibit the predominance of the proposed method in terms of subjective and objective Evaluation.

\section{Zhengguo Li, Jinghong Zheng, Zijian Zhu, and} Shiqian Wu [12] introduce a presentation combination conspire for contrastingly uncovered pictures with moving articles. The proposed plot includes a phantom evacuation calculation in a low unique range area and a specifically detail-upgraded introduction combination calculation. The proposed apparition evacuation calculation incorporates a bidirectional standardization based strategy for the location of non steady pixels and a two-round half breed technique for the remedy of nonconsistent pixels. Our detail-improved introduction combination calculation incorporates a substance versatile reciprocal channel, which removes fine points of interest from all the redressed pictures at the same time in inclination space. The last picture is blended by specifically adding the extricated fine subtle elements to a middle of the road picture that is produced by melding all the redressed pictures by means of a current multiscale calculation. The proposed introduction combination calculation enables fine subtle elements to be overstated while existing presentation combination calculations don't give such an alternative. The proposed plot generally out performs existing presentation combination plans when there are moving articles in genuine scenes. What's more, the proposed apparition expulsion calculation is easier than existing phantom evacuation 
calculations and is appropriate for cell phones with constrained computational asset.

Rui Shen, Irene Cheng, Jianbo Shi, and Anup Basu [13] A solitary caught picture of a certifiable scene is generally lacking to uncover every one of the subtle elements due to under-or over uncovered areas. To tackle this issue, pictures of a similar scene can be first caught under various presentation settings and after that joined into a solitary picture utilizing picture combination methods. In this paper, we propose a novel probabilistic model-based combination procedure for multi-presentation pictures. Not at all like past multi-introduction combination techniques, our strategy plans to accomplish an ideal harmony between two quality measures, i.e., nearby difference and shading consistency, while consolidating the scene points of interest uncovered under various exposures. A summed up irregular strolls system is proposed to figure an internationally ideal arrangement subject to the two quality measures by detailing the combination issue as likelihood estimation. Analyses show that our calculation creates brilliant pictures at low computational cost. Examinations with various different procedures demonstrate that our strategy produces better outcomes as a rule.

\section{III.METHODOLOGY}

\section{A. Multiple image sequence}

The database comprises of source picture, for multi introduction picture combination a succession of pictures that is set of pictures under various presentation levels which are taken to prepare the pictures. At that point they are subjected to prehandling. These pictures at that point intertwined to get a superb melded picture, which is taken an underlying picture. The source picture does not contain much data which is taken as information and the incorporated yield picture is more educational then the information picture. For the most part the info has various pictures of same scene taken at various presentation levels. All information pictures were scaled into a few down-tested layers by utilizing the Laplacian pyramid [16]. Multi-determination changes has been perceived as the most helpful way to deal with dissect the data which contain all pictures with the end goal of picture combination. The discrete wavelet change has turned into a most helpful instrument for combination.

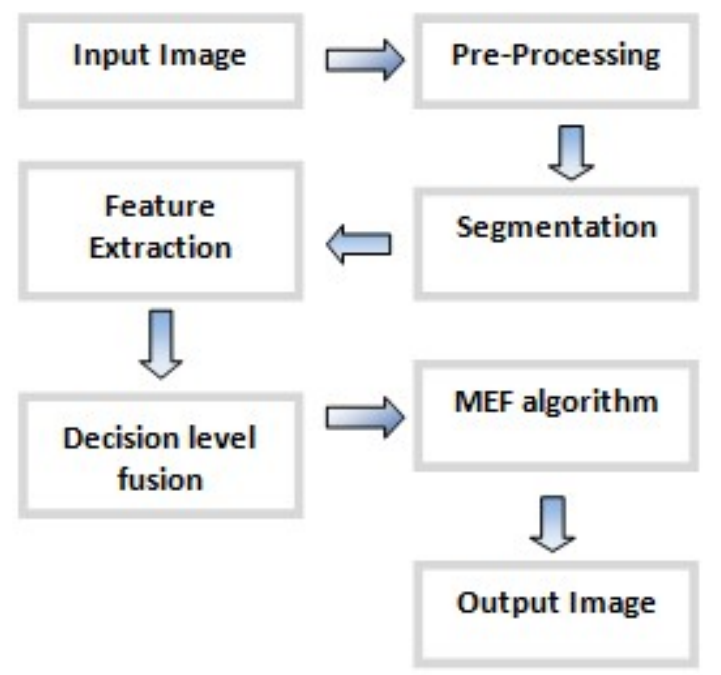

Fig1. Flowchart of the Designed system

\section{B. MEF-SSIM}

The first MEF-SSIM will bar the luminance correlation. With regards to developing $\mathrm{MEF}$ calculations, the mean force of each shading patch should be expressly said. Roused by the strategy we gauge the coveted mean power of the combined picture fix by

$$
\hat{I}=\frac{\sum_{k=1}^{K} u\left(\mu_{k}, I_{k}\right) I_{k}}{\sum_{k=1}^{K} u\left(\mu_{k}, I_{k}\right)}
$$

The construction of MEF-SSIM follows the definition of the SSIM is

$$
S\left(\left\{x_{k}\right\}, y\right)=\frac{\left(2 \mu_{\widehat{x}} \mu_{y}+C_{1}\right)\left(2 \sigma_{\widehat{x} y}+C_{2}\right.}{\left(\mu_{\widehat{x}}^{2}+\mu_{y}^{2}+C_{1}\right)\left(\sigma_{\widehat{x}}^{2}+\sigma_{y}^{2}+c_{\iota}\right.}
$$

By thinking about a substantial grouping of pictures and deciding the quality measure for every one of the picture factual techniques can be utilized to decide a general quality measure of the pressure strategy. Characterizing picture quality as far as a dissimilarity from the first circumstance, quality measure ends up specialized as in they can be unbiasedly decided regarding deviations from the first models. Picture quality albeit identified with the subjective view of a picture e.g., Human taking a gander at a photo.

\section{IV.RESULTS}

Twenty four source image sequence are selected in this work, which spread diverse scenes containing both light and dark regions with different colour occurrences. On the other side, the proposed algorithm is initialized with the fused images. These include two simple operators they are local and global energy which linearly fuse the images, they used as weighting factors denoted by LE and GE, respectively, and sophisticated ones with different 
perceptual emphasis such as Mertens09 [10], Shen11 [4], Gu12 [6], Bruce14 [9], Shen14 [7], and Ma15 $[8]$.

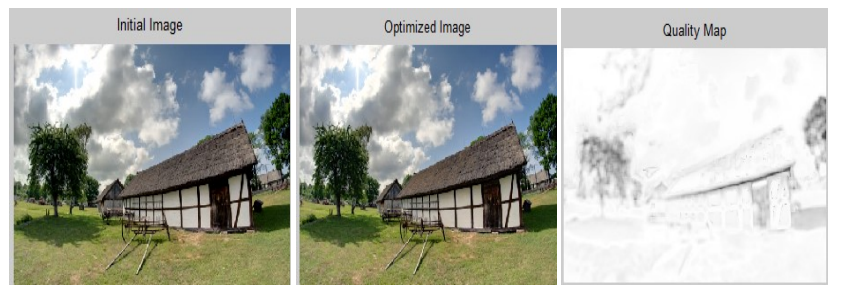

Fig2. Output Simulation results of 'Kluki' for 59 iterations

The above fig.2 explains the simulation window consists of three images: first is initial image, second is optimized image, and third is quality map. The initial image indicates sequence of images in the data base is fused and forms an initial image. The source image is fused with the test image, here with sequence of source images the test image is taken from detailed enhancement algorithm based on Mertens07[28]. It can be seen that it fails to preserve some relevant details such as the tree and the door part of the image. Those details are faithfully recovered in the MEFSSIM optimized image. Initially the fused image value is 0.9323 , at the $59^{\text {th }}$ iteration in this simulation 0.9852 value is obtained which means the image is more bright, where brighter indicates better quality.

The above fig.3 shows value of MEF-SSIM as a function of iteration on the 'Kluki' sequence using different initial images taken at different exposure levels as starting point. By observing the graph, the MEF-SSIM values increases monotonically with iterations.

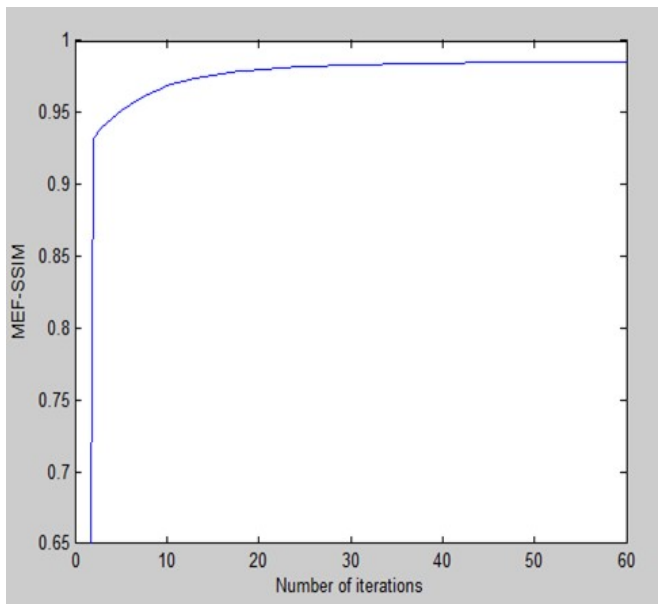

Fig3. MEF-SSIM as a function of iteration on the 'Kluki' sequence with initial fused images created by MEF algorithm

\section{CONCLUSION}

We propose an alternate way to deal with plan MEF calculations by specifically working in the space all things considered. Numerous MEF calculations include at least one free parameters in which the best qualities to a great extent rely upon the picture content. Iteratively looking for a picture that enhances MEF-SSIM which is progressed MEF picture quality evaluation display developed after existing MEFSSIM. The proposed calculation is iterative so it isn't reasonable for continuous applications. This calculation can discover neighborhood optima on the grounds that the non convexity of MEF-SSIM is exceedingly attractive. Picture combination has turned into a by and large utilized innovation to build the visual translation of the pictures in various applications like expanded vision framework, therapeutic finding, mechanical technology, military and reconnaissance. It has been usually utilized as a part of numerous fields, for example, question recognizable proof, grouping and change discovery.

\section{REFERENCES}

1. Genderen and Pohl 1994).

2. Guest editorial of Information Fusion, 2007

3. E. Reinhard, W. Heidrich, P. Debevec, S. Pattanaik, G. Ward, and K. Myszkowski, High Dynamic Range Imaging: Acquisition, Display, and Image-Based Lighting. San Mateo, CA, USA: Morgan Kaufmann, 2010.

4. Shutao Li, Member, IEEE and Xudong Kang, Fast Multi-exposure Image Fusion with Median Filter and Recursive Filter, IEEE Transactions on Consumer Electronics, Vol. 58, No. 2, May 2012.

5. E. Reinhard, W. Heidrich, P. Debevec, S. Pattanaik, G. Ward, and K. Myszkowski, High Dynamic Range Imaging: Acquisition, Display, and Image-based Lighting. Burlington, MA, USA: Morgan Kaufmann, 2010.

6. J. Im, S. Lee, and J. Paik, "Improved elastic registration for ghost artifact free high dynamic range imaging," IEEE Trans. Consumer Electronics, vol. 57, no. 2, pp. 932-935, May 2011.

7. Z. Li, J. Zheng, and S. Rahardja, "Detail-enhanced exposure fusion," IEEE Trans. Image Process., vol. 21, no. 11, pp. 4672-4676, Nov. 2012. 
8. S. Li, X. Kang, and J. Hu, "Image fusion with guided filtering," IEEE Trans. Image Process., vol. 22, no. 7, pp. 2864-2875, Jul. 2013.

9. Kede Ma, Student Member, IEEE, Zhengfang Duanmu, Student Member, IEEE, Hojatollah Yeganeh, Member, IEEE, and Zhou Wang, Fellow, IEEE, Multi-Exposure Image Fusion by Optimizing A Structural Similarity Index, IEEE transactions on computational imaging, vol. 4, no. 1, march 2018.

10. Z. Liu, E. Blasch, Z. Xue, J. Zhao, R. Laganiere, and $\mathrm{W}$. Wu, "Objective assessment of multiresolution image fusion algorithms for context enhancement in night vision: A comparative study," IEEE Trans. Pattern Anal. Mach. Intell., vol. 34, no. 1, pp. 94-109, Jan. 2012.

11. S. Li and X. Kang, "Fast multi-exposure image fusion with median filter and recursive filter," IEEE Trans. Consum. Electron., vol. 58, no. 2, pp. 626-632, May 2012.
12. Z. Li, J. Zheng, Z. Zhu, and S. Wu, "Selectively detail-enhanced fusion of differently exposed images with moving objects," IEEE Trans. Image Process., vol. 23, no. 10, pp. 4372-4382, Oct. 2014.

13. R. Shen, I. Cheng, J. Shi, and A. Basu, "Generalized random walks for fusion of multiexposure images," IEEE Trans. Image Process., vol. 20, no. 12, pp. 3634-3646, Dec. 2011.

14. K. Ma, K. Zeng, and Z. Wang, "Perceptual quality assessment for multi exposure image fusion," IEEE Trans. Image Process., vol. 24, no. 11, pp. 3345-3356, Nov. 2015.

15. Shutao Li, Xudong Kang, and Jianwen $\mathrm{Hu}$, "Image Fusion with Guided Filtering", IEEE transactions on image processing, vol. 22, no. 7, july 2013.

16. P. Burt and T. Adelson, "The Laplacian pyramid as a compact image code," IEEE Trans. Commun., vol. 31, no. 4, pp. 532-540, Apr. 1983. 